\title{
Erratum
}

\section{Two-Phase Terpene Total Synthesis: Historical Perspective and Application to the Taxol ${ }^{\circledR}$ Problem}

Yoshihiro Ishihara, Phil S. Baran* Synlett 2010, 1733.

The advance online publication (e-First) version of this article contained errors in Figure 3 and in the related text. Those errors have been corrected for both the current online version and the print publication. 